\section{Extranodal diffuse large B-cell lymphomas: A retrospective case series and review of the literature}

\author{
Stergios Boussios, ${ }^{1,2}$ Ioannis Zerdes, ${ }^{1,2}$ \\ Amalia Vassou, 2,3 Eleni Bareta, ${ }^{1,2}$ \\ Esmeralda Seraj, 1,2 \\ Alexandra Papoudou-Bai, ${ }^{4}$ \\ Nicholas Pavlidis, ${ }^{1,2}$ Anna Batistatou, ${ }^{4}$ \\ George Pentheroudakis ${ }^{1,2}$ \\ ${ }^{1}$ Department of Medical Oncology, \\ 2Society for Study of Clonal \\ Heterogeneity of Neoplasia (EMEKEN), \\ ${ }^{3}$ Department of Hematology, and \\ ${ }^{4}$ Department of Pathology, Medical \\ School, University of Ioannina, Greece
}

\begin{abstract}
Non-Hodgkin lymphomas commonly show extranodal involvement (25-30\%) but primary diffuse large B-cell lymphomas (DLBCL) with extranodal localization represent clinically and molecularly distinct entities. The present study involved retrospective analysis of case records of 4 patients who were diagnosed with extranodal DLBCL between 2010 and 2016 at the Medical Oncology and Hematology Departments of the Ioannina University Hospital, Greece. Median age of presentation was 69 years (range 60-77 years). There were 2 males and 2 females. The sites of DLBCL involvement included adrenal gland, mandible, cervix uteri, and ileum. Two patients had B symptoms while none had bone marrow involvement. After staging workup, all the patients fell into $\mathrm{I}_{\mathrm{E}}$ stage. The treatment approach included chemotherapy combined with rituximab (R), whereas one patient received additionally irradiation therapy. Post-treatment positron emission tomography-computed tomography scan was performed in 3 patients. In terms of the outcome, 3 patients are alive in complete response, whereas one was lost in follow-up. Further prospective data analyses are required so as to better elucidate the biology and course of extranodal DLBCL.
\end{abstract}

\section{Introduction}

Lymphomas represent a heterogeneous group of hematological neoplasms and have been traditionally divided into Hodgkin (20-30\%) and Non-Hodgkin lymphomas (NHL, 70-80\%). The latter affect 386,000 cases worldwide per year $(2.7 \%$ of all cancers) and include both aggressive and less aggressive types of B-cell and T-cell origin. ${ }^{1}$ The most common worldwide type $(40 \%)$ of NHL is diffuse large B-cell lymphomas (DLBCL). ${ }^{2}$ Despite a common morphology characterized by the diffuse proliferation of mature large B-cells, these tumors have biological and clinical heterogeneity. Nevertheless, DLBCL predisposes lymphatic regions, $\leq 40 \%$ initially present in extranodal sites. ${ }^{3}$ In regard to medical and family history, lifestyle and predisposing factors, extranodal DLBCL may differ as compared to their nodal counterparts. ${ }^{4}$ This data in addition to the differences in molecular pathogenesis, clinical presentation and natural history, indicate that extranodal DLBCL are distinct entities. The predominant site of origin is the gastrointestinal (GI) tract; nevertheless many other organs and tissues may be involved such as the testis, central nervous system (CNS), breast, mediastinum, skin and bone.

The evaluation of disease specific epidemiological factors is highly demanding. ${ }^{4}$ CNS lymphomas are commonly related to immunodeficiency, and some studies excluding these patients have suggested that CNS DLBCL have a reverse correlation with atopic diseases and are influenced by family history of lymphoma and smoking. Testicular lymphomas have been associated with B-cell activating autoimmune diseases and smoking, whereas mediastinal DLBCL are positively related to family history of NHL, prolonged use of hair dye and living on farms. ${ }^{4}$ Excluding female gender predisposition, there is no clear epidemiological association or risk factors specifically for primary breast lymphomas.

Overall, extranodal disease with involvement of tissue other the lymph node, spleen, Weldeyer's ring, and thymus is not common among DLBCL. They are frequently not diagnosed because their symptoms are similar to many other diseases of inflammatory etiology. During differential diagnostics procedure all diseases which are characterized by lymphadenopathy, such as toxoplasmosis, mononucleosis, lymphadenitis, metastases and Hodgkin lymphoma, must be considered. Immunochemotherapy is a reasonable and tolerable therapeutic approach. At the present time, surgery has not proven to be routinely effective. In extranodal lymphoma, surgical resection of the lesion and radiotherapy are used, whereas in aggressive lymphomas, combined local treatment with complementary or inductive radiotherapy or chemotherapy is applied.
Correspondence: George Pentheroudakis, Department of Medical Oncology, Medical School, University of Ioannina, Greece, Stavros Niarchou Avenue, 45110, Ioannina, Greece.

Tel./Fax:+30.26510.99394

E-mail:gpenther@otenet.gr

Key words: Chemotherapy; Extranodal; Lymphomas; Immunohistochemistry.

Acknowledgements: Dr. Boussios is clinical research fellow funded by the Medical School of the University of Ioannina, Greece.

Contributions: the authors contributed equally.

Conflict of interest: the authors have declared no potential conflict of interest.

Received for publication: 28 January 2017.

Revision received: 15 April 2017.

Accepted for publication: 18 April 2017.

This work is licensed under a Creative Commons Attribution-NonCommercial 4.0 International License (CC BY-NC 4.0).

CCopyright S. Boussios et al., 2018

Licensee PAGEPress, Italy

Hematology Reports 2018; 10:7070

doi:10.4081/hr.2018.7070

\section{Materials and Methods}

In this retrospective study, we describe the clinical features, staging procedure and therapeutic outcome of 4 patients with extranodal primary DLBCL, treated in our tertiary center, where over 100 cases of localized nodal disease were managed between 2010 and 2016. All data were inputted anonymously and were dealt with in accordance with good clinical practice.

To define the neoplasm extension, workup included bone marrow aspirate, trephine biopsy and CT scan of the head and neck region, brain, thorax, abdomen, and pelvis. Histopathologic diagnosis was made according to the criteria of the World Health Organization (WHO) Classification of Tumors of Hematopoietic and Lymphoid Tissues. ${ }^{5}$ Additional immunohistochemical stains were applied in all cases. Immunohistochemistry examination included analysis of Ki 67 (proliferative) index and the mouse monoclonal antibodies directed against CD79a, CD20, CD5, CD30, CD10, CD3, CD2, CD45, ALK, cyclin D1, CLA, IRF4/MUM1, BCL-2 and BCL-6. Ann Arbor system was used to classify patients according to their stage.

Response was determined by International Workshop Response Criteria 
(IWRC) and PET/CT criteria. Post-treatment PET/CT scan was performed in 3 among the 4 patients. Tumor response was evaluated according to the RECIST, version 4.0.

\section{Results}

\section{Patients' characteristics}

Patients' characteristics and demographics are depicted in Table 1. The median age at diagnosis was 69 years (range, 60 to 77 years). The gender distribution for the entire population was 2 males to 2 females. Immunohistochemical analysis of the specimens revealed a diversity in the expression of various markers, with CD20 to be positive in all cases while the Ki67 marker had an expression of above $70 \%$ in all available specimens. According to the Hans classification, in our case series 1 patient $(25 \%)$ had a germinal center and $3(75 \%)$ a nongerminal center profile. ${ }^{6}$

The sites of involvement included adrenal gland, mandible, cervix uteri, and ileum. The clinical presentation depended on the location of the disease. One patient suffered from abdominal pain (small intestine site of disease), while vaginal bleeding, and swollen mandible were present at the cervix uteri, and mandible location respectively.

Two patients had B symptoms (50\%), while none had bone marrow involvement. After staging workup, all the patients fell into $\mathrm{I}_{\mathrm{E}}$ stage. The majority of patients had low International Prognostic Index (IPI) and as a result they are considered of good prognosis.

\section{Management}

The initial treatment included surgery for 3 out of four patients $(75 \%)$, while immunochemotherapy was delivered to all of them. One patient was treated with a combination of chemotherapy and radiotherapy (Involved-Field Radiation Therapy - IFRT). Most patients $(\mathrm{n}=3,75 \%)$ received
R-CHOP (cyclophosphamide, doxorubicin, vincristine and prednisolone), whereas 1 patient was treated with R-CEOP (replacement of doxorubicin with epirubicin).

After the completion of the treatment, the 3 patients who examined with PET/CT, achieved CR according to PET/CT criteria, while one participant was lost to follow up. Table 1 summarizes the therapeutic choices and the best responses to the treatment.

\section{Discussion and literature review}

The median age at diagnosis for DLBCL is in the sixth and seventh decades. Immunosuppressive conditions and viruses such as Epstein-Barr virus (EBV), Human Immunodeficiency virus (HIV) and hepatitis $\mathrm{C}$ virus (HCV) have been associated with the pathogenesis of DLBCL. ${ }^{4}$ However, in this retrospective analysis, this was not the case, as there was no evidence of immunosuppression in our cohort of patients. There is a slight male preponderance overall, but this varies among anatomic sites. DLBCL typically produces large, destructive lesions that may invade adjacent structures. Microscopic examination reveals a diffuse proliferation of large cells with round, oval, irregular, or lobated nuclei, distinct nucleoli, and scant cytoplasm. The definition of primary extranodal NHL is still controversial and this may con- tribute to explain their varying percentage in comparison with the nodal ones. In the general population, extranodal NHL comprise 24 to $48 \%$ of all NHL. ${ }^{7}$ There has been a distinction between primary DLBCL, presenting de novo, and secondary DLBCL. In most cases, patients present with localized disease. The designation of stage III and IV lymphomas as primary extranodal NHL is indeed questionable since extranodal involvement in the presence of mainly nodal or disseminated disease may represent secondary extranodal disease spread.

In the present study, we have attempted to report and evaluate the clinical and pathological features as well as response to therapy and survival in patients diagnosed with extranodal DLBCL. Of most interest is that only approximately $4 \%$ of patients diagnosed with DLBCL involve extranodal region, based on the data collected from the electronic patient records of the local University Hospital. The majority of the DLBCL cases (three out of four) were of the centroblastic morphologic variant; the remainder was of the immunoblastic variant, whereas anaplastic variants were not identified (Figure 1). There were no major differences between the characteristics of these patients as compared to the literature; nevertheless, the incidence of primary extranodal DLBCL is relatively rare in this

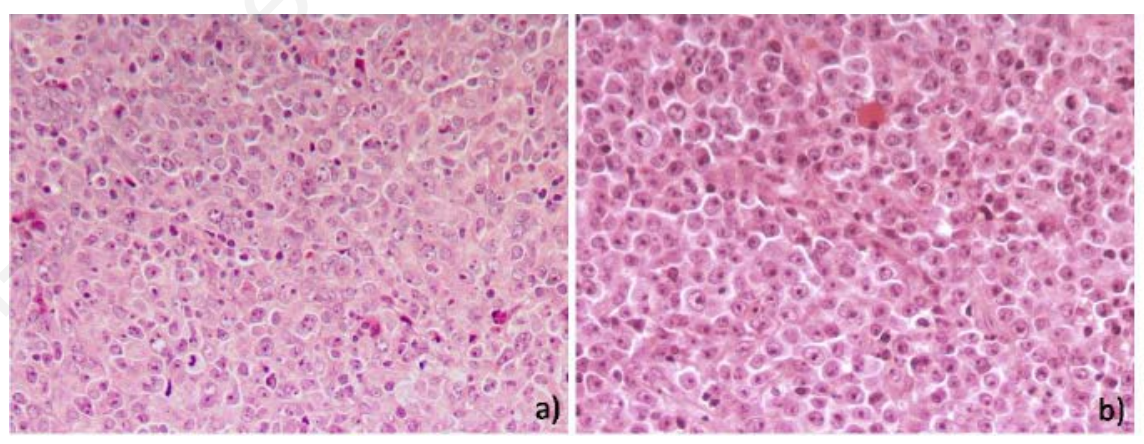

Figure 1. Representative images of morphologic variants of diffuse large B-cell lymphomas in the current study (Hematoxylin and Eosin stain, magnification $\times 400$ ). Centroblastic (a); Immunoblastic (b).

Table 1. Demographics of patients with extranodal diffuse large B-cell lymphomas.

\begin{tabular}{|c|c|c|c|c|c|c|c|c|c|}
\hline Case & Age & Gender & Site & Symptoms & Stage & $\begin{array}{l}\text { Year of } \\
\text { treatment }\end{array}$ & $\begin{array}{l}\text { Treatment } \\
\text { given }\end{array}$ & $\begin{array}{l}\text { Response } \\
\text { status }\end{array}$ & Present \\
\hline 1 & 77 & M & Adrenal gland (right) & Fever, weight loss & $\mathrm{I}_{\mathrm{EB}}$ & 2010 & $\begin{array}{l}\text { R-CEOP21 } \\
\times 6\end{array}$ & CR & Alive in CR \\
\hline 2 & 69 & M & Mandible & Swelling of the mandible & $\mathrm{I}_{\mathrm{E}}$ & 2012-2013 & $\begin{array}{c}\mathrm{R}^{-\mathrm{CHOP}_{21}} \\
\times 4\end{array}$ & NA & NA \\
\hline 3 & 60 & $\mathrm{~F}$ & Cervix uteri & Abdominal vaginal bleeding & $\mathrm{I}_{\mathrm{E}}$ & 2013 & $\begin{array}{l}\mathrm{R}-\mathrm{CHOP}_{21} \\
\times 6+\text { IFRT }\end{array}$ & CR & Alive in $\mathrm{CR}$ \\
\hline 4 & 70 & $\mathrm{~F}$ & Small intestine (ileum) & Abdominal pain, weight loss & $\mathrm{I}_{\mathrm{EB}}$ & 2014 & $\begin{array}{c}\mathrm{R}^{-\mathrm{CHOP}_{21(2 / 3)}} \\
\times 4+2 \mathrm{R}\end{array}$ & CR & Alive in CR \\
\hline
\end{tabular}

DLBCL: Diffuse Large B-cell Lymphomas; M: Male; F: Female; R: Rituximab; CEOP: Cyclophosphamide, Epirubicin, Vincristine and Prednisolone; CHOP: Cyclophosphamide, Doxorubicin, Vincristine and Prednisolone; CR: complete response; NA: not available 
study probably because this group of disorders is supported by various specialties related to the location of lymphoma in our center. The incidence of primary extranodal presentation is variable across the different B-cell histologic subtypes, encompassing the majority of Burkitt's lymphomas, up to $50 \%$ of DLBCL and less than $10 \%$ of follicular lymphomas (FL). ${ }^{8}$ Overall, primary extranodal DLBCL usually affect GI tract, CNS, bone, skin and non-lymphatic structures of head and neck. Various classifications have been proposed based on molecular, pathologic and phenotypic characteristics, taking into consideration the heterogeneity of this entity. ${ }^{3}$ The histological subtype is undoubtedly the main predictor of prognosis in either nodal or extranodal lymphomas, and the response to therapy varies among patients. Other prognostic factors include age, performance status, stage, tumor burden and/or extra nodal localization. This is partially due to differences in natural history, but mainly to differences in management strategy which are related to organ-specific problems. In general, data on the prognostic value of extranodal sites is limited; though, our small case series indicates that the extranodal DLBCL has potential prognostic value in the $\mathrm{R}$ era. Indeed, in our institution they have been associated with a better prognosis, as compared to nodal DLBCL which is in accordance with the literature. Markers that are correlated with a more aggressive behavior of the disease are Lactate Dehydrogenase (LDH), $\beta$ 2 microglobulin levels and proliferation rate. The IPI and age-adjusted IPI (aaIPI) have been used to stratify patients in different high or low-risk subgroups and thus allowing clinicians to modify their therapeutic strategies. ${ }^{9}$

Cure rates are high in patients with limited disease (5-year PFS of $80-85 \%$ ) whereas a 5-year PFS of approximately $50 \%$ can be noticed in patients with advanced disease. The treatment of choice is CHOP chemotherapy combined with the monoclonal anti-CD20 antibody R, nevertheless in some cases chemoradiation therapy offers better outcome. In our case series, 3 patients received $\mathrm{R}-\mathrm{CHOP}$ regimen chemotherapy whereas R-CHOP combined with IFRT was administered in 1 patient respectively, which is in accordance to the European Society for Medical Oncology (ESMO) guidelines. ${ }^{10}$ Despite improvements in survival after the introduction of $\mathrm{R}$, a proportion of patients still remains refractory or relapses. New treatments targeting specific tumor alterations are currently under evaluation. NF- $\mathrm{NB}$ activation and resistance to chemotherapy is a wellknown molecular pathway that could be overcome by these novel therapies. ${ }^{11}$ At that regard, MYD88 pathway could block the viability of tumor cells. Indeed, bortezomib, a proteasome inhibitor, can act synergistically with R-CHOP inhibiting NF- $\kappa \mathrm{B}$ and improve the outcome of post-germinal/activated DLBCL patients. DLBCLs carrying MYD88 mutations could be particularly sensitive to those drugs inhibiting either NF- $\kappa$ B or JAK/STAT signaling pathways. ${ }^{11}$

The role of PET/CT scan in DLBCL is crucial for treatment response assessment, ${ }^{12}$ and was predominantly implemented in our cases (3 out of 4 patients). In that regard $\mathrm{PET} / \mathrm{CT}$ is more accurate than contrastenhanced CT scan, with increased sensitivity for nodal and extranodal sites; in practice, contrast-enhanced CT scan is often carried out before PET/CT. Indeed, CT scan may be necessary for a better delineation of lymphadenopathy from the bowel.

The pronounced heterogeneity in the pathogenesis, clinicopathological features and outcome of the various primary extranodal DLBCL underline the bounden need for detailed consideration of the different sites of origin. By examining each case separately we can delineate the similarities or differences between our series and others previously reported. On the basis of our study, extranodal DLBCL sites had a lower proportion of advanced disease at diagnoses when compared with exclusively nodal disease. A potential explanation for this finding is that involvement of extranodal sites could be detected earlier based on the symptoms associated with mass effect from the tumor.

There are obvious limitations to this study, including its retrospective nature, the absence of follow-up in one of the reported cases, the small size of the cohort, the characteristics of a single institution and the lack of MYD88 mutational status analysis that could help to recognize subgroups of patients with particular features and outcome. Future studies should focus on the potential pathological, molecular, and genetic differences between nodal and extranodal DLBCL looking for potential therapeutic targets.

\section{Primary adrenal lymphoma}

Primary adrenal lymphoma PAL is an extremely rare disorder, accounting for less than $1 \%$ of all NHL and only $3 \%$ of primary extranodal lymphomas. ${ }^{13}$ Since 2013 less than 200 cases have been published in the literature. The most common characteristics of PAL include bilateral involvement of adrenal gland, male predominance and elderly age. Symptoms of adrenal insufficiency may present especially in bilateral adrenal involvement. The most prevalent histology is DLBCL. In the largest study of PAL con- cerning 31 DLBCL, CR and overall response rate after $\mathrm{R}-\mathrm{CHOP}$ chemotherapy were $54.8 \%$ and $87.0 \%$ respectively. ${ }^{13}$ The 2-year estimates of OS and PFS were $68.3 \%$ and $51.1 \%$. In patients achieving CR, significant prolongations of OS $(\mathrm{P}=0.029)$ and PFS ( $\mathrm{P}=0.005)$ were detected. Ann Arbor stage did not affect OS. Bilateral versus uniteral involvement of adrenal gland did not impact OS. When staging was modified to include bilateral adrenal involvement as one extranodal site, early stage (I, II) was associated with longer $\operatorname{OS}(\mathrm{P}=0.021)$ and PFS $(\mathrm{P}<0.001)$.

The prognosis of PAL is dismal with 1year OS rate of up to $17.5 \%$, probably because optimal treatment lacks consensus. ${ }^{13}$ In earlier trials of CHOP therapy, OS of $20 \%-50 \%$ has been reported. The therapeutic approach include combination chemotherapy and radiotherapy, while surgical resection is not recommended due to high morbidity and mortality rates. The risk of CNS relapses is frequent; nevertheless, rituximab has significantly improved the outcome. Generally, it is rather unclear whether treatment with R-CHOP is associated with improved outcome. A few reports demonstrated some benefit in this setting. ${ }^{14}$

\section{Mandible}

Among the NHL that occur in the oral cavity, $15-45 \%$ arise in the maxilla and mandible and the most common locations are the upper jaw maxilla $(11 \%)$, the mandible $(8 \%)$, the palatal soft tissue $(8 \%)$ and the gum $(7 \%)$. In addition, NHL of the head and neck in patients with HIV presents frequently as large local masses, with destruction of the maxilla, mandible, and bones around the paranasal sinus. ${ }^{15}$ A retrospective review stated that, 16 out of 100 patients $(16 \%)$ with head and neck extranodal NHL, presented with jaw bone involvement, predominantly with DLBCL. The mandible was the most common bone affected. ${ }^{16}$ The median age at diagnosis is 64 years, and men are slightly more susceptible than women. ${ }^{17}$

Jaw tumor symptoms, may help in early detection and treatment. Rapid local destruction of the bone without obvious odontogenic infection is a key characteristic of jaw tumors. ${ }^{17}$ Persistent pain and tooth mobility caused by rapid bone destruction may be an early indication of malignancy. In advanced cases, swelling, pain, lip numbness and pathologic fracture are also associated with a primary bony lesion. ${ }^{17}$

Histopathologically, osteoclasts form tunnels penetrating the cortex and the tumor spread out from the marrow to the surrounding soft tissue through these cortical tunnels. ${ }^{18}$ Immunohistochemistry is crucial 
to reach the correct diagnosis in clinically suspicious cases.

Radiographic features are quite specific for diagnosis. Panoramic images are valuable investigation particularly at an early stage of diagnosis, allowing the detection of the first signs of malignancy such as cortical destruction, teeth extrusion and periodontal widening. Imaging may reveal pathological fracture or osteolysis with or without associated condensation of neighboring soft tissue or periosteal thickening. Widening of the mandibular canal and mental foramen may considered as findings suggestive of NHL. ${ }^{19}$ Extension the tumor beyond the bony wall in the absence of bone destruction is important in differentiating NHL from squamous cell carcinoma of the maxillary sinus. Magnetic resonance imaging (MRI) is recommended investigation in terms of the evaluation of the progress of the lesion in the soft tissues, which fails at high resolution $\mathrm{CT} .{ }^{20}$ In addition, the presence of extraglandular involvement, cervical lymphadenopathy, and the relationship to vital structures are also evaluated with imaging. ${ }^{21}$

Differential diagnosis of the tumor of the mandible should include multiple myeloma, Ewing sarcoma, Langerhans cell histiocytosis, leukemia, osteosarcoma, bone metastasis, osteomyelitis ${ }^{22}$ and can mimic inflammatory lesions that arise around the teeth apices such as periapical granuloma and radicular cyst. ${ }^{23}$

Beal et al. ${ }^{24}$ in a long-term follow-up of 82 patients with primary bone lymphoma, have concluded that patients' prognosis is excellent, with a 5-year survival rate of $95 \%$. Nevertheless, DLBCL of the mandible is usually aggressive, associated with rapid growth and severe bone destruction. ${ }^{17}$ In a retrospective analysis, only 3 out of 7 patients with primary extra nodal NHL of the oral cavity, especially DLBCL, who received chemoradiation therapy were alive in a median follow-up of 21 months. ${ }^{25}$ Furthermore, a patient with DLBCL of the mandible treated with $\mathrm{CHOP}$ chemotherapy was without signs of recurrence in a median follow-up of 18 months. $^{26}$

\section{Uterine lymphoma}

Despite the increasing incidence of NHL, only $1-1.5 \%$ arises from female genital organs. In a series of 147 isolated genital tract NHL apportionment percentage was as follows: $59 \%$ ovarian, $15.5 \%$ uterine corpus, $11.5 \%$ uterine cervix, $7.5 \%$ vulval and $6 \%$ vaginal. The remaining cases involved more than one organ. ${ }^{27}$ In up to $80 \%$ of cases, uterine and cervical NHL appear to be the primary site of disease. ${ }^{27}$ As the most common subtype of cervical NHL is
DLBCL, obviously, the lymphoma cells in smears typically appear as discohesive cells with scant cytoplasm, indistinct cellular membranes, irregular nuclear contours, one or more large prominent nucleoli, and irregular clearing of the nuclear chromatin. Lack of mucosal involvement, and sparing of stroma and uterine junctional zone seem to be unique characteristic. FL is the second most common histologic subtype of cervical lymphoma.

There are 60 cases of primary cervical lymphoma described in the literature. ${ }^{28}$ The median age of presentation is 46 years (range 20-85 years), and DCBCL account for $37 \%$ of cases. The Ann Arbor stage at presentation was I in $69.2 \%$, II in $22.7 \%$ and III and above in $8.1 \%$ of patients respectively. Treatment approach included surgery in $42 \%$ of cases, chemotherapy in $16.8 \%$, radiotherapy in $10.9 \%$, and multimodal treatment in the remainder of these patients. Rituximab was applied in $11.7 \%$ of the cohort. In $85.2 \%$ of patients there was no evidence of recurrence within a median follow up time of 40.5 months (range 2-240 months). Relapse is verified in $2 \%$ of the population within $12-48$ months, while $8.6 \%$ died of their disease within $0-40$ months.

The therapeutic approach to the rare isolated genital tract NHL is individualized and based on the general principals of NHL treatment. Non-aggressive asymptomatic NHL can be managed with surveillance or radiotherapy in the case of nodal involvement. Symptomatic patients with stage III and IV FL are treated with rituximab alone or in combination with standard chemotherapy regimes, such as CHOP. The role of surgery seems to be limited. Recently, immuno-chemotherapy regimens have established efficacy while, immunotherapy alone and conservative approach for asymptomatic early stages represent challenging modality. At that regard, successful pregnancies following treatment have been reported in the literature. ${ }^{29}$

Prognosis of cervical lymphoma appears to be dismal in advanced stage disease, but its correlation with the different histological types is not completely clarified. ${ }^{28}$

\section{Small intestinal lymphoma}

The prevalence of GI in lymphoma is relatively common accounting for $20-40 \%$ of all extranodal disease and $10-15 \%$ of all NHL. Stomach is the most common site of involvement $(60-75 \%)$ followed by the small bowel, ileum, cecum, colon and rectum. Age at diagnosis depend on the histological subtype of lymphoma. Clinical symptoms are indistinguishable from other digestive system disorders and include intermittent abdominal pain, nausea, vomiting, weight loss and rarely acute obstructive symptoms, perforation or diarrhea. ${ }^{30}$

Histopathologically, almost $90 \%$ of the primary GI lymphomas are of DLBCL with very few T-cell lymphomas and Hodgkin lymphoma. Predilection sites according to the histological subtypes have been detected and include mucosa-associated lymphoid tissue (MALT) lymphoma in stomach and mantle cell lymphoma (MCL) in terminal ileum and jejunum appearing as numerous polyps, hence called multiple lymphomatous polyposis. ${ }^{31}$ Furthermore, enteropathyassociated T-cell lymphoma (EATL) in jejunum, often complicated by Crohn's disease and FL in duodenum with a geographic variation in its distribution have been identified. ${ }^{32}$ Multifocality, however, has been noticed particularly in MALT lymphoma and FL. Multiple risk factors have been recognized in the pathogenesis of GI lymphoma including virological, immunological and clinical parameters, such as HIV, EBV, hepatitis B virus (HBV), human T-cell lymphotropic virus-1 (HTLV-1), as well as campylobacter jejuni and helicobacter pylori infection and inflammatory bowel and celiac disease either. ${ }^{33}$

Capsule endoscopy and double-balloon technique of push-and-pull enteroscopy, significantly increased the diagnostic yield in small intestinal lymphoma. Diffuse mucosal thickening and edema with multiple shallow ulcerations, and a nodular or mosaic mucosal pattern are typically present. ${ }^{34}$ Radiographic features in barium studies are not specific and include polypoid form, multiple nodules, infiltrative form, endoexoenteric form with excavation and fistulization, and mesenteric invasive form with an extraluminal mass.

The treatment outcome of intestinal lymphoma is relatively poorer than that of gastric lymphoma depending on their histologic subtypes. Lymphoma primarily located in the small intestine usually requires diagnostic laparotomy with the affected segment removed for therapeutic reasons either. The role of adjuvant therapy after curative resection for stage $\mathrm{I}_{\mathrm{E}}$ and stage $\mathrm{II}_{\mathrm{E}}$ lymphomas is controversial. There is no consensus recommendations for the treatment of MALT lymphoma involving the small intestine with various modalities depending on the disease burden and the clinical parameters. The surgical resection was traditionally considered as the main therapeutic approach in cases of local intestinal lymphomas. Multi-agent chemotherapeutic strategy has been necessitated for advanced stage intestinal lymphoma with multifocal presentation of MALT lym- 
phoma. Surveillance is a reasonable approach for indolent FL at stage IE is recommended by some authors until evidence of disease progression. ${ }^{35}$ Symptomatic patients, or advanced disease of FL requires surgery, chemotherapy with CHOP and/or radiotherapy. The latter is not suggested for DLBCL involving the small intestine. ${ }^{36}$ Anthracycline-based chemotherapy followed by radiotherapy is an option for advanced intestinal lymphoma not amenable to resection. Patients diagnosed with EATL and treated with anthracyclinebased chemotherapy regimens achieved better survival rates as compared to alternative treatment strategies; nevertheless, longterm survival is poor. According to a retrospective study of 31 patients, the 1- and 5year survival rates were $38.7 \%$ and $19.7 \%$, respectively. ${ }^{37}$ Another study revealed that patients treated with high-dose chemotherapy followed by autologous peripheral blood stem cell transplantation achieved significantly improved survival. This kind of treatment may be beneficial in $70 \%$ of patients with EATL. ${ }^{38}$

\section{Conclusions}

In this article we presented our experience in a cohort of patients with extranodal DLBCL. The biologic behavior, clinicopathologic traits and response to therapeutic interventions of these diverse malignancies should be elucidated in order the outcomes of these patients to be improved. Due to the fact that our knowledge stems from retrospective data, the designation of further prospective studies is needed.

\section{References}

1. Ferlay J, Soerjomataram I, Dikshit R, et al. Cancer incidence and mortality worldwide: sources, methods and major patterns in GLOBOCAN 2012. Int $\mathrm{J}$ Cancer 2015;136:359-86.

2. De Paepe P, De Wolf-Peeters C. Diffuse large B-cell lymphoma: a heterogeneous group of non-Hodgkin lymphomas comprising several distinct clinicopathological entities. Leukemia 2007;21:37-43.

3. Møller MB, Pedersen NT, Christensen BE. Diffuse large B-cell lymphoma: clinical implications of extranodal versus nodal presentation: a populationbased study of 1575 cases. $\mathrm{Br} \mathrm{J}$ Haematol 2004;124:151-9.

4. Cerhan JR, Kricker A, Paltiel O, et al. Medical history, lifestyle, family histo- ry, and occupational risk factors for diffuse large B-cell lymphoma: the InterLymph Non-Hodgkin Lymphoma Subtypes Project. J Natl Cancer Inst Monogr 2014;2014:15-25.

5. Sabattini E, Bacci F, Sagramoso C, et al. WHO classification of tumours of haematopoietic and lymphoid tissues in 2008: an overview. Pathologica 2010; 102:83-7.

6. Hans CP, Weisenburger DD, Greiner TC, et al. Confirmation of the molecular classification of diffuse large B-cell lymphoma by immunohistochemistry using a tissue microarray. Blood 2004; 103:275-82.

7. Corti M, Villafañe M, Bistmans A, et al. Primary extranodal non-hodgkin lymphoma of the head and neck in patients with acquired immunodeficiency syndrome: a clinicopathologic study of 24 patients in a single hospital of infectious diseases in Argentina. Int Arch Otorhinolaryngol 2014;18:260-5.

8. Groves FD, Linet MS, Travis LB, et al. Cancer surveillance series: nonHodgkin's lymphoma incidence by histologic subtype in the United States from 1978 through 1995. J Natl Cancer Inst 2000;92:1240-51.

9. Sehn LH, Berry B, Chhanabhai M, et al. The revised international prognostic index (R-IPI) is a better predictor of outcome than the standard IPI for patients with diffuse large B-cell lymphoma treated with R-CHOP. Blood 2007;109:1857-61.

10. Tilly H, Gomes da Silva M, Vitolo U, et al. Diffuse large B-cell lymphoma (DLBCL): ESMO clinical practice guidelines for diagnosis, treatment and follow-up. Ann Oncol 2015;26:116-25.

11. Rovira J, Karube K, Valera A, et al. MYD88 L265P mutations, but no other variants, identify a subpopulation of DLBCL patients of activated B-cell origin, extranodal involvement, and poor outcome. Clin Cancer Res 2016;22: 2755-64.

12. Barrington SF, Mikhaeel NG, Kostakoglu L, et al. Role of imaging in the staging and response assessment of lymphoma: consensus of the international conference on malignant lymphomas imaging working group. J Clin Oncol 2014;32:3048-58.

13. Kim YR, Kim JS, Min YH, et al. Prognostic factors in primary diffuse large B-cell lymphoma of adrenal gland treated with rituximab-CHOP chemotherapy from the consortium for improving survival of lymphoma (CISL). J Hematol Oncol 2012;5:49.

14. Kim KM, Yoon DH, Lee SG, et al. A case of primary adrenal diffuse large Bcell lymphoma achieving complete remission with rituximab-CHOP chemotherapy. J Korean Med Sci 2009; 24:525-8.

15. Weber AL, Rahemtullah A, Ferry JA. Hodgkin and non-Hodgkin lymphoma of the head and neck: clinical, pathologic, and imaging evaluation. Neuroimaging Clin N Am 2003;13:37192.

16. Etemad-Moghadam S, Tirgary F, Keshavarz S, et al. Head and neck nonHodgkin's lymphoma: a 20-year demographic study of 381 cases. Int J Oral Maxillofac Surg 2010;39:869-72.

17. Bugshan A, Kassolis J, Basile J. Primary diffuse large B-cell lymphoma of the mandible: case report and review of the literature. Case Rep Oncol 2015;8:451-5.

18. Hicks DG, Gokan T, O'Keefe RJ, et al. Primary lymphoma of bone. Correlation of magnetic resonance imaging features with cytokine production by tumor cells. Cancer 1995;75:973-80.

19. Buric N, Jovanovic G, Radovanovic Z, et al. Radiographic enlargement of mandibular canal as first feature of nonHodgkin's lymphoma. Dentomaxillofac Radiol 2010;39:383-8.

20. Imaizumi A, Kuribayashi A, Watanabe $\mathrm{H}$, et al. Non-Hodgkin lymphoma involving the mandible: imaging findings. Oral Surg Oral Med Oral Pathol Oral Radiol 2012;113:33-9.

21. Celebi I, Mahmutoglu AS, Ucgul A, et al. Quantitative diffusion-weighted magnetic resonance imaging in the evaluation of parotid gland masses: a study with histopathological correlation. Clin Imaging 2013;37:232-8.

22. Krishnan A, Shirkhoda A, Tehranzadeh J, et al. Primary bone lymphoma: radiographic-MR imaging correlation. Radiographics 2003;23:1371-87.

23. Avril L, Lombardi T, Ailianou A, et al. Radiolucent lesions of the mandible: a pattern-based approach to diagnosis. Insights Imaging 2014;5:85-101.

24. Beal K, Allen L, Yahalom J. Primary bone lymphoma: treatment results and prognostic factors with long-term follow-up of 82 patients. Cancer 2006; 106:2652-6.

25. Sirsath NT, Lakshmaiah KC, Das U, et al. Primary extranodal non-Hodgkin's lymphoma of oral cavity: a single centre retrospective study. J Cancer Res Ther 2014;10:945-50.

26. Kini R, Saha A, Naik V. Diffuse large B-cell lymphoma of mandible: a case report. Med Oral Patol Oral Cir Bucal 2009;14:421-4. 
27. Kosari F, Daneshbod Y, Parwaresch R, et al. Lymphomas of the female genital tract: a study of 186 cases and review of the literature. Am J Surg Pathol 2005;29:1512-20.

28. Anagnostopoulos A, Mouzakiti N, Ruthven S, et al. Primary cervical and uterine corpus lymphoma; a case report and literature review. Int $\mathrm{J}$ Clin Exp Med 2013;6:298-306.

29. Lorusso D, Ferrandina G, Pagano L, et al. Successful pregnancy in stage IE primary non-Hodgkin's lymphoma of uterine cervix treated with neoadjuvant chemotherapy and conservative surgery. Oncology 2007;72:261-4.

30. Li B, Shi YK, He XH, et al. Primary non-Hodgkin lymphomas in the small and large intestine: clinicopathological characteristics and management of 40 patients. Int J Hematol 2008;87:375-81.
31. Hirata N, Tominaga K, Ohta K, et al. A case of mucosa-associated lymphoid tissue lymphoma forming multiple lymphomatous polyposis in the small intestine. World J Gastroenterol 2007;13: 1453-7.

32. Ko YH, Karnan S, Kim KM, et al. Enteropathy-associated T-cell lymphoma: a clinicopathologic and array comparative genomic hybridization study. Hum Pathol 2010;41:1231-7.

33. Engels EA. Infectious agents as causes of non-Hodgkin lymphoma. Cancer Epidemiol Biomarkers Prev 2007;16: 401-4.

34. Bae JY, Ko BM, Min SK, et al. A case of enteropathy-type T-cell lymphoma diagnosed by small bowel enteroscopy: a perspective on imaging-enhanced endoscopy. Gut Liver 2012;6:516-9.

35. Yamamoto S, Nakase H, Yamashita K, et al. Gastrointestinal follicular lymphoma: review of the literature. J Gastroenterol 2010;45:370-88.

36. Aleman BM, Haas RL, van der Maazen RW. Role of radiotherapy in the treatment of lymphomas of the gastrointestinal tract. Best Pract Res Clin Gastroenterol 2010;24:27-34.

37. Gale J, Simmonds PD, Mead GM, et al. Enteropathy-type intestinal T-cell lymphoma: clinical features and treatment of 31 patients in a single center. J Clin Oncol 2000;18:795-803.

38. Sieniawski M, Angamuthu N, Boyd K, et al. Evaluation of enteropathy-associated T-cell lymphoma comparing standard therapies with a novel regimen including autologous stem cell transplantation. Blood 2010;115:3664-70. 